\title{
Randomized phase II study of sequential docetaxel and irinotecan with 5-fluorouracil/folinic acid (leucovorin) in patients with advanced gastric cancer: the GATAC trial
}

\author{
Michael Gubanski ${ }^{1,2}$, Anders Johnsson ${ }^{3}$, Eva Fernebro ${ }^{3}$, Lianna Kadar ${ }^{4}$, Ingegerd Karlberg ${ }^{5}$, Petra \\ Flygare $^{6}, \AA_{\text {Ke Berglund }}^{7}$, Bengt Glimelius ${ }^{2,7}$, and Pehr A. Lind ${ }^{1,2}$; on behalf of the Gastric Cancer Taxotere \\ vs. Campto Trial (GATAC) Study Group \\ ${ }^{1}$ Department of Oncology, Karolinska University Hospital at Södersjukhus, Sjukhusbacken 10, SE-118 83 Stockholm, Sweden \\ ${ }^{2}$ Department of Oncology-Pathology, Karolinska Institutet, Stockholm, Sweden \\ ${ }^{3}$ Department of Oncology, University of Lund, Lund, Sweden \\ ${ }^{4}$ Department of Oncology, University of Malmö, Malmö, Sweden \\ ${ }^{5}$ Department of Oncology, University of Umeå, Umeå, Sweden \\ ${ }^{6}$ Department of Oncology, Sundsvall, Sweden \\ ${ }^{7}$ Department of Oncology, Radiology and Clinical Immunology, University of Uppsala, Uppsala, Sweden
}

\begin{abstract}
Background. The optimal chemotherapy in patients with advanced gastric carcinoma (GC) is yet to be determined. We compared sequential administration of docetaxel and irinotecan, both in combination with infused 5-fluorouracil/leucovorin $(5-\mathrm{Fu} / \mathrm{Lv})$, and randomly assigned patients to start with either of the two.

Methods. Patients with previously untreated locally advanced or metastatic GC and with measurable lesions (response evaluation criteria in solid tumors; RECIST) were randomly assigned to start with docetaxel $45 \mathrm{mg} / \mathrm{m}^{2}$ (arm T) or irinotecan $180 \mathrm{mg} / \mathrm{m}^{2}$ (arm C) with bolus/44-h infusion of $5-\mathrm{Fu} / \mathrm{Lv}$ (day 1 every 2 weeks). After four courses, there was a prescheduled crossover to the alternative regimen for four additional courses.

Results. Eighty-one patients were randomized and 78 started treatment. Complete and partial responses were seen in $\mathbf{3 1}$ (40\%) patients after 8 weeks and in $32(41 \%)$ after 16 weeks, with similar results in both study arms. The median overall survival (OS) was 11.5 and 10.6 months in $\operatorname{arms} T$ and $C$, respectively $(P=0.3)$. The two schedules were feasible and did not differ in the overall rate of severe adverse events (SAEs). Conclusion. This is the first randomized comparison of two of the newer cytostatic drugs in GC therapy. No differences favoring either $\operatorname{arm} \mathrm{T}$ or $\operatorname{arm} \mathrm{C}$ were found with respect to response rate, OS, or toxicity. The median OS of 11 months indicates that sequential administration of the two combinations is effective and is similar to triple combinations. Thus, comparable efficacy to platinum combinations appears to be obtained with newer, less toxic regimens when given sequentially.
\end{abstract}

Offprint requests to: $\mathrm{M}$. Gubanski

Presented at: ASCO GI Cancers Symposium Orlando and Annual Meeting Chicago, January and June 2008.

Received: August 7, 2009 / Accepted: March 10, 2010
Key words Gastric cancer - Palliative treatment - Chemotherapy $\cdot$ Sequential treatment $\cdot$ Docetaxel $\cdot$ Irinotecan

\section{Introduction}

Surgery is the therapeutic mainstay in the treatment of gastric cancer (GC), and a prerequisite for cure. However, in many cases curative tumor resection is not possible at diagnosis due to either a nonresectable primary tumor or synchronous metastatic disease. In these patients, chemotherapy is frequently used with the purpose of improving quality and quantity of life. Although documented effects have been seen [1, 2], they are still limited, with median overall survival rates (OS) of up to about 10 months and no long-term survival in the most recent trials [3,4].

Randomized trials have shown that the median survival time can be prolonged by 4-6 months with chemotherapy compared to best supportive care alone $[1,5,6]$. The most optimal regimen remains, however, to be defined, although currently used combinations contain either continuous infusion 5-fluorouracil $(5-\mathrm{Fu})$ or biochemically modulated 5-Fu. In addition, the drugs cisplatin, doxorubicin or epirubicin, etoposide, and more lately irinotecan, docetaxel, and oxaliplatin have been used in various combinations [2, 5, 7]. In the routine treatment of advanced GC, combinations of two or, more recently, three cytostatic drugs are often recommended $[4,5,7]$.

Irinotecan and docetaxel have been investigated in GC both as single agents and in combinations. As single agents response rates of about $20 \%$ have been reported for both drugs $[5,8,9]$, which is comparable to the rates 
for other drugs with activity in GC. Phase II trials reported response rates of $31 \%-58 \%$ for irinotecan; a feasible toxicity profile and median OS of 5-11 months have been reported when the drug was combined with either cisplatin or 5-Fu [10-12]. A recently published phase III study, comparing a combination of irinotecan/5$\mathrm{Fu}$ with cisplatin $/ 5-\mathrm{Fu}$, reported overall response rates of $32 \%$ and $26 \%$ and median OS of 9 and 8.7 months, respectively [3]. For docetaxel, response rates of $37 \%-56 \%$ were reported in combination with cisplatin, and response rates of $28 \%-86 \%$ were reported for docetaxel in combination with 5 -Fu in phase II trials [13-19]. The recently reported phase III V-325 study compared docetaxel in combination with 5-Fu and cisplatin against cisplatin and 5-Fu. Response rates were $37 \%$ vs $25 \%$ and the median OS was 9.2 vs 8.6 months, respectively $(P=0.02)$ [4]. The triple combination resulted, however, in significantly increased toxicity.

Combinations of several drugs, thus, result in higher response rates than monotherapy, but are generally more toxic. There is a lack of convincing evidence of clinical benefit in terms of improved quality of life (QoL) when multiple drug combinations are used rather than sequential treatment, even if subgroups of patients likely benefit from the most aggressive upfront approach, e.g., for preoperative, tumor-downsizing purposes. Furthermore, following the Goldie and Coldman hypothesis, using active drugs in a predefined alternating sequence might reduce the risk of inducing drug resistance [20]. The present study was designed to explore the efficacy of two newer drug combinations given sequentially, an approach that has not previously been explored in advanced GC.

\section{Methods}

This study was a multicenter phase II trial aiming at randomizing 80 chemonaïve patients, older than 18 years, with histologically verified metastatic or locally advanced adenocarcinoma of the stomach or cardia. Prior adjuvant therapy, including chemoradiotherapy after radical surgery was allowed if finished more than 6 months before registration. Patients with central nervous system (CNS) metastases or a history of other malignancies than GC, except for curatively treated nonmelanoma skin cancer or in-situ carcinoma of the cervix or prior malignancies treated more than 5 years ago without recurrence, were not included. All patients were required to give written informed consent. The study protocol was approved by the Ethics Committee at the University of Uppsala and the regional committees of the participating hospitals.

All patients had radiologically measurable lesions according to the response evaluation criteria in solid tumors (RECIST), good performance status (WHO $\leq 2$ ), and adequate hematological, renal, and liver functions (defined as hemoglobin $[\mathrm{Hb}] \geq 100 \mathrm{~g} / \mathrm{l}$, neutrophils $($ ANC $) \geq 2.0 \times 10^{9} / 1$, platelets $\geq 150 \times 10^{9} / 1$, total bilirubin $\leq 1.25 \times$ upper normal limit (UNL), creatinine $\leq 1.25 \times$ UNL, and aspartate aminotransferase [ASAT], and alanine aminotransferase [ALAT] $\leq 3 \times \mathrm{UNL}$; in the case of liver metastases, ASAT and ALAT $\leq 5 \times \mathrm{UNL}$ ). Patients with unresolved bowel obstruction, uncontrolled Crohn's disease or ulcerative colitis, or a current history of chronic diarrhea were excluded. All hematological and radiological assessments were done within 8 days and 3 weeks prior to randomization, respectively. The patients started treatment within 10 days from randomization.

Patients were randomly assigned to start with four courses of docetaxel $45 \mathrm{mg} / \mathrm{m}^{2}$ (arm T) or irinotecan $180 \mathrm{mg} / \mathrm{m}^{2}$ (arm C) with the simplified de Gramont regimen of 5-Fu/Lv (days 1 and 2 every 2 weeks) (Fig. 1).After 8 weeks, i.e., four courses of treatment, patients switched to the alternative regimen, thus receiving an additional four courses of docetaxel ( $\mathrm{arm} \mathrm{C}$ ) or irinotecan $(\operatorname{arm~T})$ with the same 5-Fu/Lv-schedule.

Radiological evaluations were conducted by means of computed tomography (CT)scanning or magnetic resonance imaging (MRI) at baseline and after four courses ( 8 weeks), i.e., at the switch of combinations, and after eight courses (16 weeks), i.e., at the conclusion of the second drug combination.

Quality of life was measured with the European Organization for Research and Treatment of Cancer (EORTC) questionnaire QLQ-C30 at baseline and after the fourth and eighth cycles, i.e., at the conclusion of either of the alternative regimens. These data will be presented in a separate report.

Adverse events were recorded and graded according to the National Cancer Institute (NCI) common toxicity criteria (CTC) version 2.0 before each new treatment course and up to 30 days after the last study drug infusion. In case these criteria were not applicable, the event was defined as 1, mild; 2 , moderate; 3 , severe; or 4, life-threatening.

The primary aim was to explore the efficacy (objective response rate), and secondary aims included the toxicity profile, and OS and progression-free survival (PFS) of the planned sequential administration of the two drug combinations. If no complete or partial response $(\mathrm{CR}+\mathrm{PR})$ was seen in the first 9 patients in each treatment arm, indicating that the response rate would be less than $30 \%$, the trial would be closed for that treatment arm (Gehan's method phase II trials; step 1). If three or more responses were seen among the first 14 cases an additional 9 patients were to be added (Gehan's method phase II trials; step 2). It would then be possible to estimate the response rate with a stan- 


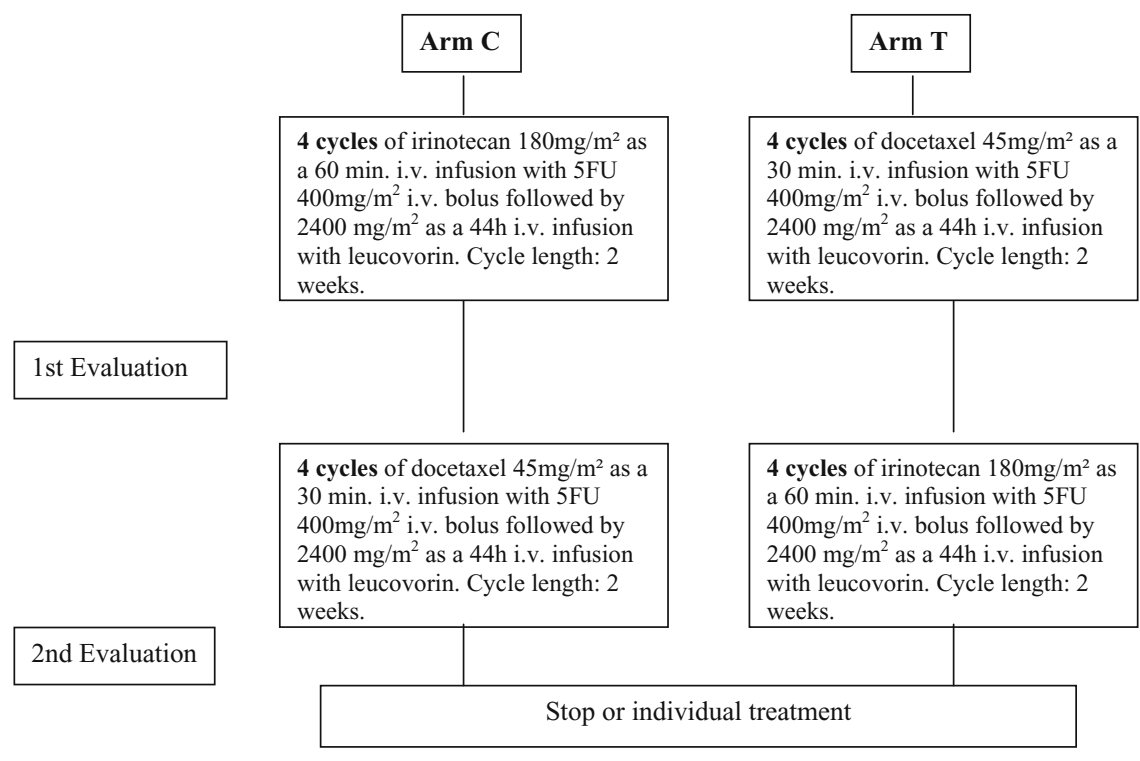

Fig. 1. Gastric Cancer Taxotere vs. Campto trial (GATAC) study design. 5FU, 5-Fluorouracil

Table 1. Characteristics of patients starting treatment

\begin{tabular}{lcc}
\hline Patients & Arm C & Arm T \\
\hline Number & 39 & 39 \\
Sex: female/male & $13 / 26$ & $5 / 34$ \\
Median age (range); years & $63(39-79)$ & $64(42-75)$ \\
Gastric surgery before study start (\%) & $16(41)$ & $17(44)$ \\
Synchronous metastases (\%) & $22(56)$ & $18(46)$ \\
Metachronous metastases (\%) & $12(31)$ & $16(41)$ \\
Distant metastases (\%) & $34(87)$ & $34(87)$ \\
Percentages of patients with involved sites 1/2/3/>3 & $13 / 54 / 28 / 5$ & $13 / 59 / 18 / 10$ \\
Percentages of patients with WHO status 0/1/2 & $44 / 44 / 1$ & $60 / 39 / 18$ \\
Median hemoglobin level at randomization (range); g/l & $120(95-156)$ & $125(80-147)$ \\
Percent elevated alkaline phosphatase at randomization (range); $\mu \mathrm{kat} / 1$ & $56(0.96-13.5)$ & $41(0.8-32)$ \\
\hline
\end{tabular}

$\mu \mathrm{kat}$, microkatal

dard error of $10 \%$. Several responses were seen in both arms in the first 9 patients. Taking into account anticipated ineligibility, inevaluability, and the second part of the study, the estimated number of patients to be enrolled per treatment arm was 40 . OS and PFS were calculated from the date of randomization and assessed according to the Kaplan-Meier method. To test for statistical significance, the $t$-test, $\chi^{2}$ test, and log-rank test were used (two-sided). A $P$ value of $<0.05$ was considered statistically significant.

\section{Results}

Eighty-one patients were randomized; 41 to arm T and 40 to arm C. One patient was diagnosed with heart failure directly after randomization and did not start any therapy. Two patients, both in arm T, withdrew their consent before the start of treatment. Thus, a total of 78 patients proceeded to treatment, i.e., 39 in each study arm. Patient characteristics are presented in Table 1. The two groups were well balanced for prognostic factors, save for gender. The number of patients treated with four and eight cycles, and reasons for dropout are shown in Fig. 2.

Seventy-one patients were evaluated for response after 8 weeks of treatment with either T or C. Of these, $31(44 \%)$ had a PR. There was no difference in objective response rates between the treatment arms (Table 2). After 16 weeks of treatment, a CR or PR as best response were seen in 2 out of $78(3 \%)$ and 30 out of $78(39 \%)$ patients, respectively, again with no difference between treatment arms. Median PFS was 4.9 months for the entire patient population and 4.9 vs 5.0 months for arms $\mathrm{C}$ and $\mathrm{T}$, respectively. Forty patients $(51 \%)$ did not have progressive disease while on the study drug. The median PFS for this subgroup was 8.1 months (range, 4-29 months).

At the time of writing, 18 months after the last patient was included, 69 patients (88\%) have died. The long- 
Table 2. Responses at first and second evaluations

\begin{tabular}{lccc}
\hline & Total $n(\%)$ & Arm T $n(\%)$ & Arm C $n(\%)$ \\
\hline $\begin{array}{l}\text { No. of patients starting treatment } \\
\text { First evaluation }\end{array}$ & 78 & 39 & 39 \\
CR & 0 & 0 & 0 \\
PR & $31(40)$ & $14(36)$ & $17(44)$ \\
SD & $24(31)$ & $13(33)$ & $11(28)$ \\
PD & $17(22)$ & $4(21)$ & $9(23)$ \\
Not evaluated & $6(8)$ & 0 & $2(5)$ \\
Second evaluation & $2(3)$ & $17(44)$ & $2(5)$ \\
CR & $30(38)$ & $10(26)$ & $5(13)$ \\
PR & $18(23)$ & $3(8)$ & $8(21)$ \\
SD & $14(18)$ & & $9(23)$ \\
PD & $8(10)$ & & $5(13)$ \\
Not evaluated & & & \\
\hline
\end{tabular}

$\mathrm{CR}$, complete response; $\mathrm{PR}$, partial response; $\mathrm{SD}$, stable disease; $\mathrm{PD}$, progressive disease

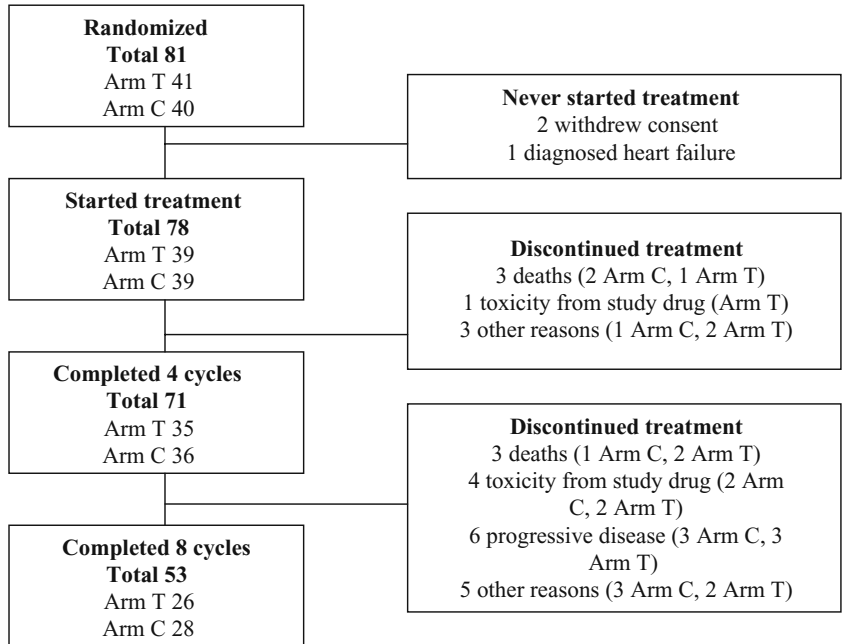

Fig. 2. Consort flowchart of patients

term survivors were evenly spread between the treatment arms, i.e., five in each arm. The survival times for these patients vary between 17 and 35 months.

The median OS was 11.5 and 10.6 months in arms T and $C$, respectively. Thus, the efficacy appears similar (log-rank test: $P=0.3$; Fig. 3 ). In this trial, there was no difference in median OS according to gender (log-rank test; $P=0.75)$.

Five patients (6\%), three in arm $\mathrm{T}$ and two in arm C, underwent surgery with curative intent after completion of chemotherapy; four had a gastrectomy and one (arm C) a deperitonealization. Four of these patients did not have distant metastatic disease at inclusion, but all had measurable disease considered nonresectable. Three of them had enlarged regional lymph nodes detectable on CT prior to treatment and one had undergone an explorative laparotomy prior to inclusion. Two of these patients are still alive at 28 and 23 months, respectively.

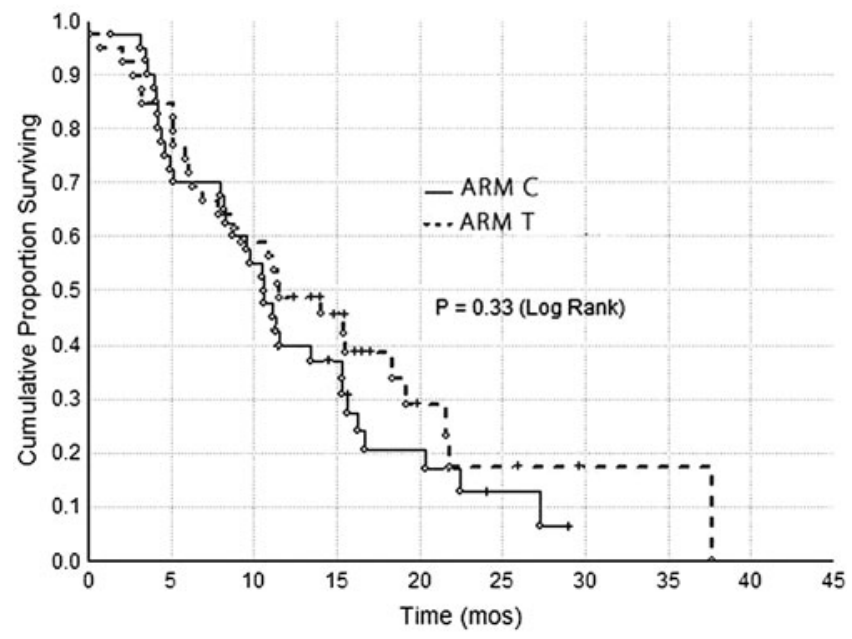

Fig. 3. Median overall survival (OS) for arms C and T. mos, Months

One patient died of recurrent GC 37 months after randomization and 21 months after surgery.

One hundred and twenty-eight grade 3 and nine grade 4 toxicities were registered. In arm T, 55 grade 3 and 6 grade 4 severe adverse events (SAEs) were observed. In arm C, 73 grade 3 and 3 grade 4 SAEs were registered. No significant difference in the overall rate of SAEs was found between the two treatment arms. The most common adverse events are listed in Table 3 . Twenty percent of the included patients experienced hematological toxicity, and $15 \%$ reported anorexia, fatigue, or infections, respectively. It can be argued whether dysphagia, which appeared in eight patients, was related to the treatment or to the tumor itself or to previous surgery. No deaths due to therapy-induced toxicity were registered.

Twenty percent of all grade 3 and $30 \%$ of grade 4 events were registered after cycles 5 to 8 . Thus, the vast 
Table 3. Most common grade 3-4 toxicities

\begin{tabular}{|c|c|c|c|c|c|c|c|c|}
\hline \multirow[b]{3}{*}{ Type of SAE } & \multicolumn{4}{|c|}{ Cycle 1-4 } & \multicolumn{4}{|c|}{ Cycle 5-8 } \\
\hline & \multicolumn{2}{|c|}{ Grade 3} & \multicolumn{2}{|c|}{ Grade 4} & \multicolumn{2}{|c|}{ Grade 3} & \multicolumn{2}{|c|}{ Grade 4} \\
\hline & Arm C & Arm T & Arm C & Arm $\mathrm{T}$ & Arm C & Arm T & Arm C & Arm T \\
\hline Infections & 5 & 8 & 0 & 0 & 0 & 1 & 0 & 0 \\
\hline Hematological toxicity & 10 & 8 & 2 & 1 & 0 & 3 & 1 & 0 \\
\hline Anorexia/dysphagia & 15 & 4 & 0 & 0 & 5 & 2 & 0 & 0 \\
\hline Nausea/vomiting & 3 & 10 & 0 & 0 & 1 & 2 & 0 & 0 \\
\hline Fatigue & 8 & 3 & 0 & 0 & 0 & 0 & 1 & 0 \\
\hline Diarrhea & 4 & 2 & 0 & 0 & 1 & 0 & 0 & 0 \\
\hline Pain & 3 & 2 & 0 & 0 & 1 & 1 & 0 & 0 \\
\hline Pulmonary embolism & 0 & 0 & 0 & 1 & 0 & 0 & 0 & 1 \\
\hline Cardiac toxicity & 0 & 0 & 1 & 0 & 0 & 0 & 0 & 0 \\
\hline Ileus & 0 & 0 & 1 & 0 & 0 & 0 & 0 & 0 \\
\hline
\end{tabular}

SAE, severe adverse event

Table 4. Recent phase III trials in advanced GC (2006-7)

\begin{tabular}{|c|c|c|c|c|c|c|}
\hline Study & Arms & $n$ & ORR (\%) & PFS (months) & OS (months) & $P$ value \\
\hline \multirow[t]{2}{*}{ V325 [23] } & $\mathrm{CF}$ & 224 & 25 & 3.7 & 8.6 & \multirow[t]{2}{*}{0.02} \\
\hline & DCF & 221 & 37 & 5.6 & 9.2 & \\
\hline \multirow{2}{*}{ V306 [3] } & IF & 168 & 32 & 5.0 & 9.0 & \multirow[t]{2}{*}{ NS } \\
\hline & $\mathrm{CF}$ & 167 & 26 & 4.2 & 8.7 & \\
\hline \multirow[t]{2}{*}{ ML17032 [24] } & $\mathrm{FP}$ & 137 & 29 & 5.0 & 9.3 & \multirow[t]{2}{*}{ NS } \\
\hline & XP & 139 & 41 & 5.6 & 10.5 & \\
\hline \multirow[t]{2}{*}{ SPIRITS [25] } & S-1 & 150 & 31 & 4.0 & 11.0 & \multirow[t]{2}{*}{0.037} \\
\hline & $\mathrm{S}-1 / \mathrm{P}$ & 148 & 54 & 6.0 & 13.0 & \\
\hline \multirow[t]{3}{*}{ JCOG 9912 [26] } & $5 \mathrm{FU}$ & 234 & 9 & 2.9 & 10.8 & \multirow[t]{3}{*}{ NS } \\
\hline & $\mathrm{CDDP} / \mathrm{I}$ & 236 & 38 & 4.8 & 12.3 & \\
\hline & S-1 & 234 & 28 & 4.2 & 11.4 & \\
\hline \multirow[t]{4}{*}{ REAL 2 [27] } & $\mathrm{ECF}$ & 263 & 41 & 6.2 & 9.9 & \multirow[t]{4}{*}{0.02} \\
\hline & $\mathrm{EOF}$ & 250 & 46 & 6.5 & 9.3 & \\
\hline & $\mathrm{ECX}$ & 245 & 42 & 6.7 & 9.9 & \\
\hline & EOX & 244 & 48 & 7.0 & 11.2 & \\
\hline
\end{tabular}

GC, gastric cancer; ORR, overall response rate; PFS, progression-free survival; OS, overall survival; JOCG, Japan Clinical Oncology Group; CF, cisplatin fluorouracil; DCF, docetaxel cisplatin fluorouracil; IF, irinotecan fluorouracil; FP, fluorouracil cisplatin; XP, Xeloda cisplatin; S-1/P, S-1 oral fluoropyrimidine cisplatin; 5FU, 5-fluorouracil; CDDP/I, cisplatin/irinotecan; ECF, epirubicin cisplatin fluorouracil; EOF, epirubicin oxaliplatin fluorouracil; ECX, epirubicin cisplatin Xeloda; EOX, epirubicin oxaliplatin Xeloda

majority of SAEs occurred during the first four treatment cycles. This could be explained partly by the fact that most events $(58 \%)$ occurred in patients who did not complete eight cycles and partly by dose reductions when toxicity was seen during the first treatment cycles. In a considerable proportion of patients, i.e., $43 \%$ of those having completed all eight cycles, no grade 3 or 4 SAEs were registered. No grade 3 or 4 SAEs were registered at follow up, 1 to 2 months after completing the eight treatment cycles.

\section{Conclusions}

This is the first randomized comparison of prescheduled sequential combination treatment in advanced GC. In addition, it allows a head-to-head comparison of early response rates and toxicity of two of the newer drugs, docetaxel and irinotecan combined with $5-\mathrm{Fu} /$ Lv. No differences favoring either docetaxel or irinotecan were found with respect to response rates or the total SAE rate after 8 weeks of therapy. Furthermore, no differences in survival outcome could be detected in relation to whether treatment was initiated with docetaxel or irinotecan. This shows that both combinations are effective in GC treatment and that they can be given safely in random order. A phase III study comparing these two treatment arms would be needed to conclude whether one of them has superior efficacy.

The main interest of our results is, however, that an objective response rate of $41 \%$ and a median OS of 11 months were reached in a multicenter study and in a population consisting of $87 \%$ of patients with distant metastatic disease. These results are similar to the recently reported efficacy data of combinations includ- 
ing cisplatin or combinations of three cytostatic agents (Table 4) [3, 4, 6, 21].

Generally, combinations of irinotecan or docetaxel with 5-Fu have been better tolerated than combinations of cisplatin and 5-Fu, but have resulted in higher gastrointestinal toxicities, with as high as $27 \%$ grade $3-4$ diarrhea for the combination of irinotecan and 5-Fu [4]. Neutropenia is a major problem for combinations of either irinotecan or docetaxel with cisplatin $(66 \%$ and $49 \%$, respectively) [4, 11]. This has specially been a problem for the TCF (docetaxel, cisplatin, 5-Fu) combination, where neutropenia occurred in $60 \%$ of the patients, resulting, notably, in a $2.5 \%$ mortality rate due to febrile neutropenia.

The toxicity of sequential irinotecan and docetaxel in our setup was relatively low if compared to the combinations mentioned above [10,18,22]. This is probably a result of "switching" the drug combination after four cycles and thus reducing the typical toxicity of each of the study drugs; i.e., hematological for docetaxel and gastrointestinal for irinotecan. Our results are encouraging, and sequential chemotherapy is a strategy well worth further investigation in the treatment of advanced $\mathrm{GC}$ and other gastrointestinal malignancies.

Another important observation in our study was of the small group of patients, with locally advanced, nonresectable tumors at diagnosis, in whom tumor reduction was obtained to such a degree that the patient could be reconsidered for surgery with curative intent. Furthermore, despite the generally poor prognosis of patients with metastatic GC, a subgroup of patients had a considerable gain in the form of comparably durable PFS and OS.

In advanced GC, metastatic disease, poor performance status, weight loss, and male gender are related to poor outcome. In the present series, no difference in OS was found according to gender; this result may have been due to the limited size of the study population.

In conclusion, the results of the GATAC trial indicate that sequential administration of the two described irinotecan and docetaxel combinations is feasible and effective, with median OS similar to those for the commonly used, more toxic, epirubicin cisplatin fluorouracil $(\mathrm{ECF}) /$ epirubicin oxaliplatin Xeloda (EOX)/Taxotere cisplatin fluracedyl (TCF)-combinations a finding which suggests that comparable efficacy can be obtained with less toxic regimens when they are given in a sequential fashion.

\section{The GATAC study group}

The GATAC trial was developed by a study group within the Swedish Society of Gastrointestinal Oncology; the study group consisted of Bengt Glimelius (PI),
Pehr Lind (co-ordinator), Peter Gunven (Department of Oncology, Karolinska University Hospital), and Ulf Bandmann (Department of Oncology, Sörmland). Coinvestigators, not appearing in the authors' list, were Peter Nygren (Department of Oncology, Uppsala), Dorte Pedersen (Department of Oncology, Karlstad), and Jörgen Hansen (Department of Oncology,Västerås).

Acknowledgments Financial support was received from the Swedish Cancer Society, the Stockholm Cancer Society, and the Sanofi-Aventis and Pfizer Pharmaceutical companies.

\section{References}

1. Glimelius B, Ekstrom K, Hoffman K, Graf W, Sjoden PO, Haglund $\mathrm{U}$, et al. Randomized comparison between chemotherapy plus best supportive care with best supportive care in advanced gastric cancer. Ann Oncol 1997;8:163-8.

2. Janunger KG, Hafstrom L, Nygren P, Glimelius B. A systematic overview of chemotherapy effects in gastric cancer. Acta Oncol 2001;40:309-26.

3. Dank M, Zaluski J, Barone C, Valvere V, Yalcin S, Peschel C, et al. Randomized phase III study comparing irinotecan combined with 5-fluorouracil and folinic acid to cisplatin combined with 5-fluorouracil in chemotherapy naive patients with advanced adenocarcinoma of the stomach or esophagogastric junction. Ann Oncol 2008;19:1450-7.

4. Van Cutsem E, Moiseyenko VM, Tjulandin S, Majlis A, Constenla $\mathrm{M}$, Boni C, et al. Phase III study of docetaxel and cisplatin plus fluorouracil compared with cisplatin and fluorouracil as first-line therapy for advanced gastric cancer: a report of the V325 Study Group. J Clin Oncol 2006;24:4991-7.

5. Wagner AD, Grothe W, Behl S, Kleber G, Grothey A, Haerting J, et al. Chemotherapy for advanced gastric cancer. Cochrane Database Syst Rev 2005;2:CD004064.

6. Webb A, Cunningham D, Scarffe JH, Harper P, Norman A, Joffe $\mathrm{JK}$, et al. Randomized trial comparing epirubicin, cisplatin, and fluorouracil versus fluorouracil, doxorubicin, and methotrexate in advanced esophagogastric cancer. J Clin Oncol 1997;15:261-7.

7. Sastre J, Garcia-Saenz JA, Diaz-Rubio E. Chemotherapy for gastric cancer. World J Gastroenterol 2006;12:204-13.

8. Farhat FS. A general review of the role of irinotecan (CPT 11) in the treatment of gastric cancer. Med Oncol 2007;24:137-46.

9. Roth AD, Ajani J. Docetaxel-based chemotherapy in the treatment of gastric cancer. Ann Oncol 2003;14(Suppl 2):ii41-4.

10. Ajani JA, Baker J, Pisters PW, Ho L, Mansfield PF, Feig BW, et al. CPT-11 plus cisplatin in patients with advanced, untreated gastric or gastroesophageal junction carcinoma: results of a phase II study. Cancer 2002;94:641-6.

11. Boku N, Ohtsu A, Shimada Y, Shirao K, Seki S, Saito H, et al. Phase II study of a combination of irinotecan and cisplatin against metastatic gastric cancer. J Clin Oncol 1999;17:319-23.

12. Sato A, Kurihara M, Matsukawa M, Shimada K, Yamazaki T, Nakamachi M, et al. Preliminary study of fortnightly irinotecan hydrochloride plus cisplatin therapy in patients with advanced gastric and colorectal cancer. Cancer Chemother Pharmacol 2001;47:380-4.

13. Einzig AI, Neuberg D, Remick SC, Karp DD, O'Dwyer PJ, Stewart JA, et al. Phase II trial of docetaxel (Taxotere) in patients with adenocarcinoma of the upper gastrointestinal tract previously untreated with cytotoxic chemotherapy: the Eastern Cooperative Oncology Group (ECOG) results of protocol E1293. Med Oncol 1996;13:87-93. 
14. Mavroudis D, Kourousis C, Androulakis N, Kalbakis K, Agelaki S, Kakolyris S, et al. Frontline treatment of advanced gastric cancer with docetaxel and granulocyte colony-stimulating factor (G-CSF): a phase II trial. Am J Clin Oncol 2000;23:341-4.

15. Ridwelski K, Gebauer T, Fahlke J, Kroning H, Kettner E, Meyer F, et al. Combination chemotherapy with docetaxel and cisplatin for locally advanced and metastatic gastric cancer. Ann Oncol 2001;12:47-51.

16. Roth AD, Maibach R, Martinelli G, Fazio N, Aapro MS, Pagani O, et al. Docetaxel (Taxotere)-cisplatin (TC): an effective drug combination in gastric carcinoma. Swiss Group for Clinical Cancer Research (SAKK), and the European Institute of Oncology (EIO). Ann Oncol 2000;11:301-6.

17. Sulkes A, Smyth J, Sessa C, Dirix LY, Vermorken JB, Kaye S, et al. Docetaxel (Taxotere) in advanced gastric cancer: results of a phase II clinical trial. EORTC Early Clinical Trials Group. Br J Cancer 1994;70:380-3.

18. Constenla M, Garcia-Arroyo R, Lorenzo I, Carrete N, Campos B, Palacios P. Docetaxel, 5-fluorouracil, and leucovorin as treatment for advanced gastric cancer: results of a phase II study. Gastric Cancer 2002:5:142-7.

19. Moehler M, Eimermacher A, Siebler J, Hohler T, Wein A, Menges $\mathrm{M}$, et al. Randomised phase II evaluation of irinotecan plus highdose 5-fluorouracil and leucovorin (ILF) vs 5-fluorouracil, leucovorin, and etoposide (ELF) in untreated metastatic gastric cancer. Br J Cancer 2005;92:2122-8.

20. Goldie JH, Coldman AJ. A mathematic model for relating the drug sensitivity of tumors to their spontaneous mutation rate. Cancer Treat Rep 1979;63:1727-33.

21. Cunningham D, Starling N, Rao S, Iveson T, Nicolson M, Coxon $\mathrm{F}$, et al. Capecitabine and oxaliplatin for advanced esophagogastric cancer. N Engl J Med 2008;358:36-46.
22. Pozzo C, Barone C, Szanto J, Padi E, Peschel C, Bukki J, et al. Irinotecan in combination with 5-fluorouracil and folinic acid or with cisplatin in patients with advanced gastric or esophagealgastric junction adenocarcinoma: results of a randomized phase II study. Ann Oncol 2004;15:1773-81.

23. Ajani JA, Moiseyenko VM, Tjulandin S, Majlis A, Constenla M, Boni C, et al. Clinical benefit with docetaxel plus fluorouracil and cisplatin compared with cisplatin and fluorouracil in a phase III trial of advanced gastric or gastroesophageal cancer adenocarcinoma: the V-325 Study Group. J Clin Oncol 2007;25:3205-9.

24. Kang YK, Kang WK, Shin DB, Chen J, Xiong J, Wang J, et al. Randomized phase III trial of capecitabine/cisplatin (XP) vs. continuous infusion of 5-FU/cisplatin (FP) as first-line therapy in patients (pts) with advanced gastric cancer (AGC): efficacy and safety results. J Clin Oncol, 2006 ASCO Annual Meeting Proceedings 2006;24(18S):LBA4018.

25. Koizumi W, Narahara H, Hara T, Takagane A, Akiya T, Takagi M, et al. S-1 plus cisplatin versus S-1 alone for first-line treatment of advanced gastric cancer (SPIRITS trial): a phase III trial. Lancet Oncol 2008;9:215-21.

26. Boku N, Yamamoto S, Shirao K, Doi T, Sawaki A, Koizumi W, et al. Randomized phase III study of 5-fluorouracil (5-FU) alone versus combination of irinotecan and cisplatin $(\mathrm{CP})$ versus S-1 alone in advanced gastric cancer (JCOG9912). J Clin Oncol, 2007 ASCO Annual Meeting Proceedings Part I 2007;25(18S): LBA4513.

27. Cunningham A, Rao S, Starling N, Iveson T, Nicolson M, Coxon F, et al. Randomized multicenter phase III study comparing capecitabine with fluorouracil and oxaliplatin with cisplatin in patients with advanced oesophagogastric (OG) cancer: the REAL 2 trial. J Clin Oncol, 2006 ASCO Annual Meeting Proceedings 2006;24(18S):LBA4017. 